\title{
Un umanista alla corte di Federico III. Il Pentalogus di Enea Silvio Piccolomini
}

Barbara Baldi

\section{(2penEdition}

Journals

Edizione digitale

URL: http://journals.openedition.org/cei/85

DOI: 10.4000/cei.85

ISSN: 2260-779X

\section{Editore}

UGA Éditions/Université Grenoble Alpes

\section{Edizione cartacea}

Data di pubblicazione: 15 octobre 2011

Paginazione: 161-171

ISBN: 978-2-84310-207-3

ISSN: 1770-9571

Notizia bibliografica digitale

Barbara Baldi, «Un umanista alla corte di Federico III. II Pentalogus di Enea Silvio Piccolomini», Cahiers d'études italiennes [Online], 13 | 2011, online dal 15 avril 2013, consultato il 26 mars 2021. URL: http:// journals.openedition.org/cei/85 ; DOI: https://doi.org/10.4000/cei.85 


\title{
UN UMANISTA ALLA CORTE DI FEDERICO III. IL PENTALOGUS DI ENEA SILVIO PICCOLOMINI
}

\author{
Barbara Baldi \\ Università degli Studi di Milano
}

Il tema del rapporto fra Enea Silvio Piccolomini, l'impero e la Germania costituisce indubbiamente un leitmotiv degli studi piccolominiani. In fondo, è lo stesso Piccolomini a suggerire, per primo, questo riferimento. Nel suo trattato epistolare dedicato proprio alla Germania, scritto fra la fine del I457 e l'inizio del I458, egli non esita infatti a presentarsi come un "cardinale tedesco", e, rivolgendosi in particolare al cancelliere dell'arcivescovo di Magonza, Martin Mair, che lo aveva accusato di essere complice del papato e di voler depredare la chiesa e la nazione tedesca delle sue ricchezze e dei suoi benefici, il Piccolomini gli ricorda senz'altro la sua lunga permanenza in Germania, prima presso il concilio di Basilea e poi direttamente al servizio di Federico III, e i molteplici servizi che, come segretario e come ambasciatore, egli ha così reso alla nazione germanica, fino a rivendicare, per questa via, un legame privilegiato con l'impero:

Nos in ipso iuvente nostre flore Germaniam intravimus. Fuimus in concilio Basiliensi pluribus annis neque aliter nos gessimus, quam si nati educatique in ea provincia fuissemus. Ad imperatorem deinde Federicum transivimus, cui supra quindecim annos famulati sumus. Secretarium et consiliarium apud eum gessimus. Interfuimus vestris conventibus, qui de re publica gerebantur. Secreta vestra summa fide tractavimus, negotia diligenter absolvimus. Legationes quam plures obivimus, nihil nobis antiquius fuit, quam nationi vestre nostra obsequia consecrare. Neque ista dicimus tanquam improperantes. Habemus enim gratias Theutonice genti, que, cum nostra opera usa est, nobis magis quam sibi consuluit. Honoravit enim personam nostram, dum exercuit, nosque, qui prorsus iacebamus, erexit, ita ut intelligamus non nostris meritis, que scimus quam sint exigua, sed Germanico favore, et imperatorie maiestatis intuitu dignitatem cardinalatus nobis esse delatum ${ }^{\mathrm{I}}$.

I. Aeneas Silvius, Germania (und Jacob Wimpfeling: Responsa et replicae ad Eneam Silvium), a cura di A. Schmidt, Köln-Graz, Böhlau Verlag, 1962, p. 36; e, per le accuse del Mair, si v. la sua lettera al Piccolomini, 
Dunque sì, il Piccolomini, l'impero, la Germania... Ma da dove viene questo orientamento? A riconsiderare gli studi, direi che sono stati posti anzitutto in rilievo tre motivi: la tradizione imperiale, la tradizione ghibellina, la formazione umanistica.

Il primo motivo, come accennato, è quello della tradizione imperiale. La lunga esperienza del Piccolomini al concilio di Basilea si conclude nel segno del fallimento. Il Piccolomini giunge a Basilea nel I432, e, negli anni successivi, egli segue da vicino lo svolgimento dell'attività conciliare. Tuttavia, lentamente, la sua iniziale fiducia nel concilio, nel suo ruolo, cede il posto ad un senso di delusione crescente per l'incapacità dei padri conciliari di affrontare in modo efficace il problema dello scisma e della riforma della Chiesa, ed è proprio in questo ambito che matura, che maturerebbe, il richiamo all'impero. Di fronte all'inconcludenza del concilio, infatti, la ricerca della pace e dell'unità della Cristianità può essere trovata per il Piccolomini solo attraverso il ricorso ad un'autorità superiore - quella imperiale appunto, l'unica capace di porre fine al prolungarsi dello scisma e dello scontro fra il papato e il concilio. Questa rinnovata fiducia nell'impero fa così tutt'uno con il recupero - più o meno "aggiornato" - della tradizione universalistica (o medievale) dell'impero come punto di riferimento centrale della Cristianità, e, in simile luce, la figura del Piccolomini è stata anzi più volte paragonata a quella di un "novello Dante", di un "novello Petrarca".

La decisione del Piccolomini nell'autunno I442 di abbandonare il concilio di Basilea e l'antipapa Felice V per seguire Federico III rientra, rientrerebbe, a sua volta, in questa prospettiva ${ }^{2}$. Ma a questo elemento si

datata Aschaffenburg, 3I.8.I457, ivi, p. 9-IO. Le accuse del Mair, in particolare, si riferiscono ad una riserva generale concessa al Piccolomini dal pontefice per i benefici vacanti in tre province tedesche e rientrano in un attacco più generale contro la rapacità e l'avidità della Chiesa romana, in una prospettiva che si richiama direttamente alle più recenti iniziative dell'opposizione antipapale tedesca guidata allora proprio dall'arcivescovo di Magonza. Ma per un esame più diretto del legame fra la lettera del Mair, il problema più generale del rapporto fra la chiesa di Roma e la nazione tedesca, e la composizione della Germania, mi permetto di rimandare a B. Baldi, Pio II e le trasformazioni dell'Europa cristiana, Milano, Unicopli, 2006, p. 3I-50. - Per la nomina a cardinale del Piccolomini, avvenuta nel dicembre del I456, in riferimento al rapporto con l'impero si v. M. Pellegrini, "Pio II, il Collegio cardinalizio e la Dieta di Mantova», in A. Calzona, F. P. Fiore, A. Tenenti, C. Vasoli (a cura di), Il sogno di Pio II e il viaggio da Roma a Mantova (Atti del convegno internazionale, Mantova, I3-I5 aprile 2000), Firenze, Olschki, 2003, p. 26-27 e, ivi, R. Fubini, «Conclusioni», p. 593. - Il Piccolomini, come spiego subito dopo nel testo, giunge a Basilea nel I432, al seguito del cardinal Domenico Capranica, diventa nel I 438 membro del concilio e poi segretario dell'antipapa Felice V, all'inizio del I44O. Nel I442 lascia il concilio ed entra al servizio di Federico III, dove rimane fino al I455, quando, inviato a Roma dall'imperatore come ambasciatore presso il neo-eletto Callisto III, si ferma nella penisola, continuando però a mantenere stretti legami con il mondo imperiale. Per un primo orientamento sulla biografia piccolominiana, si v. comunque M. Pellegrini, "Pio II», in Enciclopedia dei papi, Roma, Treccani, 2000, pp. 663-685.

2. Per una lettura in questo senso si v. soprattutto F. Battaglia, «Il pensiero politico di Enea Silvio Piccolomini», in Id., Enea Silvio Piccolomini e Francesco Patrizi: due politici senesi del Quattrocento, Siena, Istituto comunale 
aggiunge il secondo motivo, quello della tradizione ghibellina, che, nel caso del Piccolomini, è di derivazione sia familiare che cittadina.

La famiglia del Piccolomini ha infatti dietro di sé una lunga tradizione ghibellina 3 ; la città di Siena si riconosce da sempre come alleata dell'impero 4; e anche il Piccolomini, in una famosa lettera del 1437 all'imperatore Sigismondo di Lussemburgo, riprende direttamente questo tema, contrapponendo senz'altro Siena a Firenze, città guelfa, nemica per eccellenza dunque dell'impero e anzi usurpatrice delle prerogative imperiali in Toscana:

Florentiam dico, que est Guelforum caput. Intelligis, quid Guelfi nomine significetur? Guelfus est, qui resistit imperium, Gibellinus, qui paret. Sed que magis imperio civitas rebellis est, quam Florentia, que magis emula? Lecte sunt noviter in concilio littere, quibus Florentini nullum se dominum recognoscere jactitant. Scis, cum Romam peteres, quo te vultu exceperint, scis quibus te Ferrare muneribus visitarint. Sed heu, decus imperii! Non sufficit Florentinis imperium abnegare, imperiales etiam occupant civitates, quas non ad jugum, sed ad exterminium subjiciunt, ut testimonio est Aretium et splendor ille quondam Tuscie Pisana civitas, nunc ruina et vile equorum stabulum, cujus instar modo etiam redigenda infelix Lucana civica obsidetur. Inde ad urbem tuam Senensem prelium transferetur ${ }^{5}[\ldots]$.

Accanto a questi due elementi, c'è poi il terzo motivo, la formazione umanistica, che per il Piccolomini ha un rilievo essenziale, tanto che egli è addirittura indicato negli studi come l' "apostolo dell'umanesimo in Germania”,

di arte e storia, 1936, pp. 3-71; T. Buyken, Enea Silvio Piccolomini. Sein Leben und Werken bis zum Episkopat, Bonn-Koln, L. Röhrscheid, I93I; J. B. Toews, «Dream and Reality in the Imperial Ideology of Pope Pius II», Medievalia et Humanistica, no I6, 1964, pp. I-73; Id., "The View of Empire in Aeneas Silvius Piccolomini (Pope Pius II)", Traditio, no XXIV, I968, pp. 47I-487.

3. Si v. in particolare R. Mucciarelli, Piccolomini a Siena. XIII-XIV secolo. Ritratti possibili, Pisa, Pacini ed., 2005; P. Pertici, «Il viaggio del papa attraverso il territorio senese: il viaggio di una vita», in Il sogno di Pio II, cit., pp. I43-I62. Sul tema si v. comunque anche R. Ceserani, «Pio II", in I protagonisti della storia universale, V. Milano, CEI, I966, p. I69-196; A. Esch, «Enea Silvio Piccolomini als Papst Pius II: Herschaftspraxis und Selbstdarstellung», in H. Boockmann, B. Mőller, K. Stackmann (a cura di), Lebenslehren und Weltentwürfe im Übergang vom Mittelalter zur Neuzeit (Abhandlungen der Akademie der Wissenschaft im Gottingen, Philhist. Kl., Dritte Folge 179), Gottingen, Vandenhoeck \& Ruprecht, 1989, pp. II5-II6; B. Widmer, Enea Silvio Piccolomini. Papst Pius II. Biographie and ausgewählte Texte aus seinen Schriften, Basel-Stuttgart, Benno Schwabe \& Co., I960, p. I4; e, della stessa autrice, Enea Silvio Piccolomini in der sittlichen und politischen Entscheidung, Basel-Stuttgart, Helbing \& Lichtenhahn, 1963, pp. I36-137.

4. Si v. per un primo orientamento P. Pertici, a cura di, Tra politica e cultura nel primo Quattrocento senese. Le epistole di Andreoccio Petrucci (I426-I443), pref. di R. Fubini, Siena, Accademia senese degli Intronati, I990.

5. Enea Silvio Piccolomini all'imperatore Sigismondo di Lussemburgo, [Basilea], 15.6.I437, in R. Wolkan, Der Briefwechsel des Eneas Silvius Piccolomini, Wien I909-I9I8, Abt. I, Briefe aus der Laienzeit (43I-I445), Bd. I, Privatbriefe, ep. 25, qui p. 77-78 (d'ora in poi solo Wolkan), corsivi miei. — L'attacco contro Firenze mira in particolare a dissuadere l'imperatore dall'accettare la proposta, sostenuta anche da Eugenio IV, della scelta della città toscana come sede per il possibile concilio di unione con la chiesa greca, tema sul quale in quel momento si stava concentrando il dibattito all'interno del concilio e fra il concilio e il papa. Per un primo riferimento a questi avvenimenti resta ancora utile il lavoro di N. Valois, Le pape et le concile. I4I8-I450. La crise religieuse du XV siècle, II, Paris, Picard, I909. 
insistendo, in questo senso, sul valore culturale (o culturale-religioso) della sua "missione" e sul richiamo alla tradizione civile e cristiana dell'Europa che a questa si accompagna ${ }^{6}$. In questa prospettiva, l'incoronazione a poeta del Piccolomini, avvenuta per mano di Federico III nel luglio I442, assume un'importanza senz'altro centrale? ${ }^{7}$. L'incoronazione è la prima che si svolge in Germania ${ }^{8}$. Essa, in particolare, nasce nell'ambito dei contatti personali del Piccolomini con alcuni membri della corte regia - il cancelliere Gaspare Schlick, il vescovo di Chiemsee, Silvestro Pflieger, l'arcivescovo di Treviri, Jacob von Sierck ${ }^{9}$ - e rappresenta per lui un riconoscimento di rilievo, cui egli tiene e cui resterà a lungo legato ${ }^{10}$ : il titolo di "poeta" risveglia echi che riportano alla tradizione classica, all'esempio, ancora una volta, di Petrarca, all'umanesimo ${ }^{\text {If; }}$ corrisponde al modo particolare in cui egli intende il rapporto fra politica e cultura. E, in effetti, l'importanza del ruolo avuto dal

6. Si v. ora su questo tema soprattutto J. Helmrath, "Vestigia Aeneae imitari” Enea Silvio Piccolomini als "Apostel" des Humanismus. Formen und Wege seiner Diffusion», in J. Helmrath, U. Muhlack, G. Walther, a cura di, Diffusion des Humanismus. Studien zur nationalen Geschichtsschreibung europäischer Humanisten, Göttingen, Wallstein Verlag, 2002, pp. 99-I4I. Si cfr. anche F. J. Worstbrock, «Piccolomini Aeneas Silvius», in Die deutsche Literatur des Mittelalters. Verfasserlexikon, Bd. 7, Berlino-New York, Walter de Gruyter, 1989, cols. 660-665.

7. L'incoronazione avviene il 27 luglio I442: si v. il diploma edito in Enee Silvii Piccolominei postea Pii PP. II, Carmina, a cura di A. v. Heck, Città del Vaticano, Biblioteca Apostolica Vaticana, 1994, pp. 215-2I6.

8. Si cfr. K. Schottenloher, «Kaiserliche Dichterkrönungen im Heiligen Römischen Reiche deutscher Nation", in A. Brackmann (a cura di), Papsttum und Kaisertum. Forschungen zur politischen Geschichte und Geisteskultur des Mittelalters. Festschrift Paul Kehr, Monaco, Verlag der Münchner Drucke, 1926, pp. 227-255; e la voce "Dichterkrönung» in Lexicon des Mittelalters, v. III, Monaco-Zurigo, Artemis Verlag, 1986, pp. 975-977.

9. L'incontro fra questi personaggi e il Piccolomini avviene nell'estate del I442, in occasione della dieta di Francoforte indetta per definire la possibile posizione della nazione germanica di fronte allo scisma ormai apertosi fra il concilio, l'antipapa e il pontefice Eugenio IV. Il Piccolomini, in particolare, era presente in qualità di membro dell'ambasciata inviata dall'antipapa Felice $V$ per presenziare all'incoronazione regia del neo-eletto Federico III. La proposta della nomina a poeta del Piccolomini matura in questa occasione: in particolare, essa nasce da un'iniziativa del vescovo di Chiemsee, ed è appoggiata presso Federico III sia dallo Schlick che dal von Sierck. Su queste tre figure, con vari riferimenti al loro rapporto con il Piccolomini e al ruolo da essi avuto, si v. P.-J. Heinig, Kaiser Friedrich III. (I440-I493). Hof, Regierung und Politik, I, Köln, Weimer, Wien, Böhlau Verlag, 1997, pp. 638-646 (sullo Schlick); pp. 58I-584 (sul Pflieger); pp. 635-638 (sul von Sierck). Si v. inoltre Buyken, Enea Silvio Piccolomini, cit., pp. 42-46; A. Lhotsky, Aeneas Silvius und Österreich, BaselStuttgart, Helbing \& Lichtenhahn, 1965, pp.I2-I3; W. Baum, «Enea Silvio Piccolomini und Österreich», in ID. (a cura di), Konziliarismus und Humanismus. Kirchliche Demokratisierungsbestrebungen im spätmittelalterlichen Österreich, Wien, Turia und Kant, 1996, pp. 75-77. Sul ruolo del Chiemsee, si v. anche la voce relativa a c. di G. Westermaher, in Allgemeine Deutsche Biographie, Bd. 25, Berlino, Duncker und Humbolt, p. 342. — Sul tema si v. anche più in generale D. Mertens, «Zu Sozialgeschichte und Funktion des poeta laureatus im Zeitalter Maximilians I», in R. C. Schwinges, a cura di, Gelehrte im Reich: zur Sozial-und Wirkungsgeschichte akademischer Eliten des I4. bis 16. Jahrhunderts, Berlino, Duncker \& Humblot, 1996, pp. 327-348; e, ivi, A. Sottili, «Ehemalige Studenten italienischer Renaissance-Universitäten: ihre Karrieren und ihre soziale Rolle», pp. 4I-74.

Io. Si cfr. Enea Silvio Piccolomini a Gaspare Schlick, Innsbruck [dicembre I442], Wolkan, Abt. I, Bd. I, ep. 43. Come risulta dalle sue lettere, nel corso dei due anni successivi il Piccolomini firmerà le proprie epistole usando il titolo di "poeta”.

II. Si v. in particolare G. Paparelli, Enea Silvio Piccolomini. L'umanesimo sul trono di Pietro, Ravenna, A. Longo Ed., 1978 (ma prima ed. 1950), p. 68. - Sull'incoronazione a poeta di Francesco Petrarca come modello per le lauree poetiche successive, conferite dal potere papale e/o imperiale, si v. soprattutto O. Kristeller, «Il Petrarca 
Piccolomini nella diffusione dell' umanesimo in ambito tedesco - in Austria, in Boemia, in Germania - è stata più volte sottolineata. La maggior parte delle sue opere, del suo epistolario, sono destinati ad un pubblico tedesco. Entrato nella cancelleria imperiale, ben presto egli diventa il referente principale di una rete di "umanisti" tedeschi che si viene man mano raccogliendo intorno a lui e che vede, tra le sue figure principali, personaggi di spicco della corte e della cancelleria imperiale, come Procopio von Rabstein, Wilhelm von Stein, Giovanni Eich... ${ }^{\text {I2 }}$

Questo impegno del Piccolomini, d'altra parte, ha sì un valore culturale, ma con una sensibilità e una finalità politiche. Il Piccolomini mantiene un senso fortissimo infatti dell'identità cristiana e civile dell'Europa, e lì, in quell'ambito, l'impero ha un ruolo centrale, di ordine, di sicurezza. La partecipazione del Piccolomini alla politica ecclesiastica di Federico III, la sua azione a favore del riavvicinamento fra il sovrano, la nazione tedesca e la chiesa di Roma, fino alla conclusione del concordato di Vienna del I448, e poi ancora negli anni successivi, si misurerebbero anche su questo sfondo particolare ${ }^{13}$.

I tre motivi che ho richiamato - la tradizione imperiale, la tradizione ghibellina, la formazione umanistica - convergono dunque tutti nell'indicare l'esistenza di un rapporto privilegiato fra il Piccolomini, l'impero, la Germania, offrendo indicazioni e riferimenti sicuramente importanti.

nella storia degli studi», in L. Rotondi Secchi Tarugi (a cura di), Petrarca e la cultura europea, Milano, Nuovi Orizzonti, 1997, pp. 7-29.

I2. Così, ad es., il von Rabenstein, funzionario della cancelleria regia dal 1437 e poi cancelliere del regno di Boemia, è il destinatario del trattato epistolare sulla Fortuna (Vienna, 26.6.I444, Wolkan, Abt. I, Bd. I, ep. I5I); al von Stein - consigliere del duca Alberto VI d'Austria e membro della corte regia - sono indirizzati la lettera in difesa della poesia (Vienna, I.6.I444, ivi, ep. I44) e il De cura et natura equorum (Vienna, 4.7.I444, ivi, ep. 154); mentre al von Eich è indirizzato il De curialium miseriis, (Bruck a M., 30.II.I444, ivi, ep. I66). — Si v. comunque, più in generale, Helmrath, “"Vestigia Aeneae imitari”», cit.; M. Palumbo, «"Teutonicus Eneas”. Aspetti della fortuna tedesca di Enea Silvio Piccolomini», in Nymphilexis. Enea Silvio Piccolomini, l'umanesimo e la geografia, (Catalogo della mostra a cura di C. Crescentini, L. Guerini), Roma, Edizioni dell'associazione culturale Shakespeare \& Company2, 2005, pp. 20I-227. Mi permetto inoltre di rimandare a B. Baldi, «La corrispondenza di Enea Silvio Piccolomini dal I43I al I454. La maturazione di un'esperienza fra politica e cultura», Reti medievali Rivista, IX, 2009 (I confini della lettera. Pratiche epistolari e reti di comunicazione nell'Italia tardomedievale, Atti della giornata di studi, Isernia, 9 maggio 2008, a cura di I. Lazzarini), on line sul sito www. retimedievali.it.

I3. Si v. in questo senso Paparelli, Enea Silvio Piccolomini, cit.; E. Garin, «Ritratto di Enea Silvio Piccolomini», in Id., Ritratti di umanisti, Milano, Bompiani, 1996 (ma prima ed. 196I), pp. 9-39; J. G. Rowe, «The Tragedy of E. S. Piccolomini (Pope Pius II): an interpretation», Church History, XXX, 196I, pp. 290-293. Il concordato di Vienna (I448) pone fine alla posizione di neutralità assunta dalla nazione tedesca nei confronti dello scontro fra il concilio, l'antipapa e la chiesa di Roma, sancendo il ritorno della Germania all'obbedienza romana e aprendo nello stesso tempo la via per la definitiva conclusione dello scisma della Chiesa (I449). Il Piccolomini, a sua volta, segue da vicino queste trattative, partecipando, come inviato di Federico III, alle varie diete tedesche di questi anni e impegnandosi direttamente in alcune importanti missioni diplomatiche a Roma, e continuando, anche dopo il I448, a porsi come un punto di riferimento centrale nelle relazioni fra la curia romana e la corte imperiale. 
Tuttavia, mi sembra, essi rischiano di far dimenticare la fatica, le incertezze, i dubbi che pure caratterizzano questo rapporto, questa lunga vicenda tedesca del Piccolomini. Al momento del suo passaggio dal concilio di Basilea alla corte di Federico III, la Germania, il mondo imperiale - questo non va dimenticato - restano, infatti, un mondo nuovo, una realtà nuova, che sì il Piccolomini in parte conosce, o pensa di conoscere, che apprezza, cui per certi versi si sente anche legato, ma che egli coglie per ora solo parzialmente ${ }^{14}$.

Da questo punto di vista, a mio avviso, l'esperienza tedesca e imperiale del Piccolomini si configura piuttosto come una scoperta: una scoperta contrastata, a tratti difficile, e che comporta, via via, nel corso di questi anni, fra il I442 e il I455, una serie ininterrotta di aggiustamenti, di verifiche, e un rapporto mutevole con la realtà che lo circonda. A partire, se si vuole, dal problema più diretto del suo inserimento in questa realtà. Quando, nell'autunno del I442, il Piccolomini decide di seguire Federico III e di diventare segretario regio ${ }^{15}$, egli può contare, certo, su alcuni presupposti importanti - il peso delle sue connessioni personali, la fiducia nell'impero, il richiamo alla propria tradizione ghibellina, l'umanesimo. Tuttavia, il passaggio non è così scontato e lo stesso inserimento del Piccolomini all'interno della corte, il problema del suo ruolo e del suo rapporto con la politica regia e con la realtà tedesca sono ancora tutti da verificare, da definire.

I4. La esperienza compiuta dal Piccolomini fra il I432 e il I442 può aver alimentato il senso di una sua certa familiarità con il mondo tedesco. Nel I432 egli entra infatti al servizio di Nicodemo della Scala, vescovo di Frisinga e rappresentante al concilio del duca Alberto VI d'Austria, e lo segue alla dieta di Francoforte dell'ottobre dello stesso anno. Nel I438 è a Vienna, dove accompagna il vescovo di Novara, Bartolomeo Visconti, nella sua missione diplomatica presso Alberto II d'Austria, appena eletto re di Germania. Negli anni successivi, partecipa, questa volta a nome di Felice $\mathrm{V}$ o del concilio, a varie missioni diplomatiche, che lo portano ora a Strasburgo, ora a Costanza, ora, di nuovo, a Francoforte, nel I442 - dove avviene, come ricordato, l'incontro con Federico III da cui nascerà poi la decisione di lasciare Basilea per la corte imperiale. Tuttavia, questa familiarità resta al dunque abbastanza relativa, e lascia soprattutto aperto, come cerco di spiegare nel testo, il problema del confronto diretto del Piccolomini con la realtà tedesca, con i suoi molteplici problemi e le sue diverse connessioni. Ma per un riesame più diretto dell'esperienza tedesca del Piccolomini in questo senso mi permetto di rinviare a B. Baldi, Esperienza tedesca e visione dell'impero in Enea Silvio Piccolomini, Tesi di dottorato in Storia Medievale, relatori proff. G. Chittolini, B. Vigezzi, Università degli Studi di Milano, 2007. Si cfr. anche B. Baldi, «La Boemia ussita in una lettera di Enea Silvio Piccolomini a Giovanni Carvajal (2I.8.I45I): fra esperienza diplomatica e riflessione storico-politica», in B. Baldi, M. M. Benzoni, (a cura di), Lontano da dove. Sensazioni, aspirazioni, direzioni, spazi fra Quattrocento e Seicento, Milano, Unicopli, 2009, pp. 13-40.

15. La proposta di entrare al servizio di Federico nasce, ancora una volta, da un'iniziativa del vescovo di Chiemsee, sempre nel corso della dieta di Francoforte dell'estate del I442. Il Piccolomini, dopo un primo momento di esitazione, accetta, e, a metà novembre, lascia definitivamente Basilea al seguito di Federico III: si v. le indicazioni già offerte qui n. 9. Il Piccolomini presta giuramento come segretario della cancelleria imperiale nel gennaio del '43: si v. Baum, Enea Silvio Piccolomini, cit., pp. 76-77. 
Proprio questo tema è, in fondo, al centro del Pentalogus ${ }^{16}$. Il Pentalogus è la prima opera che il Piccolomini compone come segretario di Federico III. Essa è infatti scritta fra il febbraio e il marzo del '43, poche settimane dopo il suo arrivo nella cancelleria regia, in corte, ed è costruita come un dialogo immaginario cui partecipano non solo Federico III e il Piccolomini, ma anche alcuni di quei personaggi cui si è accennato: il vescovo di Chiemsee, Silvestro Pflieger; e il cancelliere Gaspare Schlick, oltre al vescovo di Frisinga, Nicodemo della Scala, presso il quale il Piccolomini aveva prestato servizio alcuni anni prima ${ }^{17}$.

Il testo, in particolare, è stato considerato a lungo dagli studi ora come la conferma dell'adesione da parte del Piccolomini alla tradizione e all' ideologia imperiale ${ }^{\mathrm{r}}$; ora piuttosto come un'opera essenzialmente letteraria, legata alla missione di "apostolo dell'umanesimo" del Piccolomini in Germania ${ }^{19}$. Questi elementi, certo, sono sì presenti; ma sono inseriti nell'ambito dell'esperienza concreta che il Piccolomini fa della corte, della realtà tedesca.

L'avvio del trattato, in questo senso, colpisce. Il Pentalogus si apre in effetti sull'incontro fra il Piccolomini e Federico III, un incontro che nasce un po' per caso, quando Federico III lo vede quasi nascosto in un angolo, e lo chiama a sé, chiedendogli, un po' sorpreso, se lui sia quello stesso Enea che egli ha incoronato poeta e che è entrato al suo servizio, e domandandogli quindi perché lo abbia visto sinora così raramente ${ }^{20}$. Il Piccolomini ha però subito la risposta pronta, e lo scambio di battute che ne nasce è indicativo delle difficoltà che egli, appena giunto in corte, si trova ad affrontare, e, insieme, della sua volontà di trovare un proprio spazio di espressione.

Da un lato, infatti, la sua figura di "poeta e umanista" sembra restare piuttosto estranea alla realtà della corte, della sua organizzazione, tanto che il Piccolomini, rispondendo ai rimproveri di Federico III, si lamenta di

I6. Eneas Silvius Piccolomini, Pentalogus, ed. a cura di C. Schingnitz, Hannover, Hahnsche, 2009 (Monumenta Germaniae historica. Scriptores. Staatsschriften des späteren Mittelalters, 8), d'ora in poi semplicemente Pentalogus.

17. Per la datazione e la struttura del testo si v. Schingnitz, Einleitung, in Pentalogus, cit., pp. I-5, I6-I9. - Per il rapporto fra il Piccolomini e il vescovo di Frisinga, si v. qui n. I4.

I8. Si v. ad es. in questo senso G. Voigt, Enea Sylvius Piccolomini als Papst Pius der Zweite und sein Zeitalter, Berlin, G. Reimer, I856-I863 (rist. anast. Berlin 1967), I, pp. 304-307; Buyken, Enea Silvio Piccolomini, cit., pp. 48-49; Battaglia, "Il pensiero politico», cit., pp. 20-26; Widmer, Enea Silvio Piccolomini, cit., pp. 48-50.

19. Si v. ad es. C. M. Ady, Pius II. The Umanist Pope, London, Meuthen and Co. Ltd., 1913, p. 75; Paparelli, Enea Silvio Piccolomini, cit., p. 70; e si cfr. anche G. Zippel, «Enea Silvio Piccolomini e il mondo germanico. Impegno cristiano e civile dell'umanesimo", La cultura, XIX, I98I, pp. 296-300. - Per un riesame complessivo della storiografia sul Pentalogus si v. comunque ora anche Schingnitz, Einleitung, cit., pp. 5-I2.

20. Pentalogus, p. 56-58. 
non riuscire a ricevere udienza perché non inserito in nessuno dei gruppi di funzionari della corte:

Aut tua est aut ostiariorum culpa. Venio dietim ad fores, ianuam nunc pedibus, nunc manibus insulto frustra. Interdum supplex intromitti oro. "Mi ianitor », inquam, "sine me regem visere. Curialis sum, ceseris servus sum ». Surdus est, non audit. Quasi Ulixes adversus Sirenarum cantus ceratas aures habet. Nec Latine ago secum. Pauca hec Teutonice scio et, cum deficio, puerum summitto. Forsitan illi sic imperatum est. Ingreditur pincerna, cocus, auceps, villicus, etyops, fricator equorum, canum altor. Solus poeta et tuus, ut dicis, secretarius in limine exclusus manet vel tedio affectus recedit. Nunquam michi conspectus datur tuus, nisi cum vulgo licet tumque non affatus, sed visus tantum permittitur ${ }^{2 \mathrm{I}}$.

Dall'altro lato, nello stesso tempo, il modo in cui egli intende il suo ruolo di segretario si rivela subito diverso e ben più ampio di quello che il sovrano gli propone. Il Piccolomini infatti non intende limitarsi al semplice compito di colui che deve scrivere lettere composte da altri ${ }^{22}$, e, di fronte alle obiezioni di Federico III, rivendica senżaltro il suo valore, le sue possibilità, fino a proporsi, in quanto poeta $e$ segretario, come un sorta di consigliere privilegiato accanto ai membri del consiglio regio:

Oculatum te fore, mi rex, et non, quod ante pedes modo est, videre, sed etiam illa, que futura sunt, prospicere non inficior. Consiliarii quoque tui et prudentissimi sunt et, quod ab illis maxime exigendum est, amantissimi honoris tui. Si tamen fateri vis verum, sepe unus videt, quod alii non vident, nec ambigo, quin tibi usu aliquando evenerit, ut extra consiliariorum tuorum numerum unus aliquis et item alter ad res gerendas magnum lumen prebuerit. Quod si et michi aliquando in mentem veniret, quod utile tibi esset, non vis, id tibi ut communicatum faciam ${ }^{23}$ ?

Il Pentalogus si sviluppa da qui, e, in questo senso, senza indicare un progetto politico vero e proprio - come qualche volta si è pensato - corrisponde piuttosto al desiderio, alla volontà del Piccolomini di costruirsi, di rivendicare concretamente un proprio spazio di partecipazione, di azione personale, in una prospettiva che si lega in pari tempo alla vigorosa affermazione dell'importanza della cultura umanistica - di cui egli si sente orgoglioso portavoce - come presupposto indispensabile dell'azione politica.

Il poeta infatti - come il Piccolomini spiega a Federico III - non è solo colui che scrive poesie, ma assume un compito ben più ampio, quello cioè di mostrare come ben vivere, attraverso la condanna dei vizi e l'esaltazione

2I. Ivi, p. 58.

22. Così infatti Federico III ricorda al Piccolomini quali sono i limiti del suo ruolo di segretario: "Communem utilitatem haud tibi esse cure oportet, nisi ut epistolas dictes, cum tibi committitur": ivi, p. 6o.

23. Ivi, p. 6o. Si v. anche le osservazioni di Schingnitz, Einleitung, cit., p. 4-5, I2-15. Si cfr. inoltre Heinig, Kaiser Friedrich, cit., I, p. I69. 
delle virtù, e in particolare, egli insiste sulla funzione persuasiva che la poesia, come l'oratoria, possono avere, anche rispetto alla vita politica:

Quia poetam me creasti, hoc suspicaris, quasi nil habeat poeta preter carmina et, ut creatus poeta est, vir desinat esse. Quod etiam si verum esset, non tamen contempnendi poete forent [...] Videsne poetam vite cursum ostendere et que amanda sint et que vitanda docere? At tu solis confessoribus et capellanis animam reris tradenda. Sed frustra, crede, illos habes, nisi, qui forment animum honestis vite preceptis, habeas, quod longe melius poeta faciat quam confessor oportet, cum nemo vitia relinquat, nisi persuasus. Persuadere autem aut poete est aut oratoris. De republica vero an audiendi poete sint aliquid dicentes, paucis agam exemplis $[\ldots]^{24}$.

Gli esempi, fra gli altri, di Solone, di Tirteo, il riferimento alla figura di Sigismondo di Lussemburgo, di Alfonso d'Aragona, rafforzano questa visione:

Atque ut pretereamus antiquiores, Sigismundum tu ipse nosti. Nam et sepe in eius presentia fuisti et ille te aliquando, si viveres, imperialem sedem habiturum profatus est. Illum, quia per se loquebatur etiam in rebus magnis, omnes collaudabant, tantoque mirabantur magis, quanto rem magis obsoletam diligentius instaurare nitebatur. Beatum se quisque putabat illum alloquens, cum responsum imperialis oris audiret. Non hic tutore indiget aut curatore, dicebant. Facit hoc idem nostra in etate Alfonsus Aragonum rex, quod sibi non minus prodest quam vires exercitus. Quod unum et si tu, cesar, assequaris, ut te audiant, qui ad te veniunt, inferiores omnes te erunt principes unusque predicaberis toto in orbe integer princeps. Namque cum humanitate, clementia, pietate, religione et iustitia ceterisque animi virtutibus alios vincas, hac una locutionis dote vinci te pati non debes, in qua ceteros vincimus animantes. Adhibendum igitur litteris est aliquod tempus formandusque animus doctrina. Namque si recte volumus iudicare, scientiis atque doctrinis omnia parent. $\mathrm{Nec}$ verum est tam armis quam litteris retineri imperium ${ }^{25}$.

Proprio questo richiamo all'arte della persuasione, all'oratoria - strumento essenziale della diplomazia, dell'azione diplomatica - diventa il leitmotiv del trattato. L'umanesimo è il linguaggio essenziale della diplomazia, ed è a questi due elementi - umanesimo e diplomazia - che il Piccolomini affida lo svolgimento di tutto il trattato.

Il dialogo, in particolare, assume il carattere di una sorta di consiglio immaginario, in cui il Piccolomini svolge il ruolo di interlocutore principale, chiamato a mostrare ad un riluttante Federico III le possibilità dell'azione imperiale, e il modo in cui queste dovrebbero essere sviluppate. Il dialogo è costruito ad arte, e permette al Piccolomini di superare, di rispondere alle varie obiezioni che gli sono poste, e di dimostrare così la 
sua competenza, la sua capacità oratoria, la sua conoscenza di problemi e di situazioni diverse.

Il punto di partenza della discussione è legato alla politica ecclesiastica di Federico III, ai rapporti fra mondo tedesco, concilio e papato. Il Piccolomini, in particolare, richiama la proposta, sostenuta da Federico III, del terzo concilio come possibile soluzione dello scontro fra il concilio e il papato; ricorda le decisioni prese dai principi tedeschi nella dieta di Norimberga, appena conclusa ${ }^{26}$. Ma, da qui, la discussione si arricchisce di riferimenti diversi, che rimandano più direttamente al problema della crisi dell'impero, del suo rapporto con la realtà italiana - per quel che riguarda in particolare l'organizzazione del viaggio in Italia del sovrano e la sua incoronazione imperiale, punto di partenza essenziale per il Piccolomini per la riaffermazione del potere imperiale ${ }^{27}$; oppure, ancora, riferimenti che rimandano al rapporto fra prospettiva imperiale, realtà austriaca e principi tedeschi ${ }^{28}$.

La discussione, così, si allarga, e in breve essa coinvolge il problema del carattere stesso della politica regia, dei suoi strumenti, della ricerca del personale più adeguato, in una prospettiva che consente al Piccolomini di porre in primo piano tutta l'importanza di individuare dei collaboratori che - di fronte alla crisi del diritto e della tradizione rappresentata dallo scisma, di fronte alla sterilità del dibattito dottrinale e alle incertezze del quadro politico-ecclesiastico - siano capaci di sostenere, attraverso il ricorso agli strumenti offerti dalla cultura umanistica, l'azione e la politica imperiale nel modo più efficace. Così, ad esempio, il Piccolomini riconosce senz'altro che gli sforzi finora condotti da Federico III per l'organizzazione del terzo concilio non hanno prodotto alcun risultato ${ }^{29}$, ma questo è avvenuto perché egli si è servito di persone inadeguate, di tabellarios piuttosto

26. Pentalogus, pp. IO2-I52. Di fronte al radicalizzarsi dello scontro fra il papato e il concilio di Basilea e all'elezione dell'antipapa Felice V da parte dei padri conciliari (I439), la nazione tedesca assume una posizione di neutralità, impegnandosi nello stesso tempo per la ricerca di una possibile mediazione del conflitto ecclesiastico. La proposta del terzo concilio, in questo quadro: essa, in particolare, viene ripresa al momento della sua elezione da Federico III ed è oggetto di discussione anche alla dieta di Norimberga, che si svolge nel febbraio del '43. Per un primo riferimento più diretto alla politica ecclesiastica di Federico III e dei principi tedeschi si v. in particolare W. Stieber, Pope Eugenius IV, the Council of Basel and the secular and ecclesiastical Authorities in the Empire. The conflict over Supreme authority and power in the church, Leiden, E. J. Brill, I978.

27. Pentalogus, pp. I52-250.

28. Ivi, pp. 250-308.

29. Federico III in effetti si era rivolto ad Eugenio IV e a Felice V, ma nessuno dei due pontefici aveva però voluto accogliere la sua proposta per la convocazione di un terzo concilio: "Iam factum est, nec ulla earum, quod satis sit, respondet. Dicunt patres concilii translationem hanc esse, non novum concilium, idque ad se pertinere unaque tantum in re nobis annuunt, velle scilicet se transferre ad locum nationis Germanice, maiestati nostre gratum et rebus gerendis accommodum. Eugenius vero nihil omnino respondet, ut per eius operam sperandum de pace sit. Et illi pauca dant, iste nichil” (Pentalogus, p. II2, le parole sono attribuite a Federico III). Né Federico III aveva avuto risposte più incoraggianti da parte dei sovrani europei cui aveva scritto. 
che di oratores, incapaci di scrivere lettere efficaci, di sostenere le posizioni del sovrano e di persuadere le varie forze politiche italiane ed europee con la loro abilità oratoria:

Quoniam non per oratores, sed per tabellarios eos invitasti, credens illos ad litterarum vocationem venire vel mittere. Litterarum autem parva vis est ad eos presertim, qui se pares ducunt. Littere namque mox silent, ut lecte sunt semel, tacent, mutescunt, vilescunt, nec magni vigoris putantur, nec qui eas mittit, habere rem cordi creditur, presertim cum magna est, ut hec, de qua nunc agitur. [...] Si ergo principes non habuisti per litteras, nichil miri est. Parum namque vehemens impetrator fuisti. Quod si huius rei effectum cupis, iam non litteris, sed nuntiis et oratoribus agito. [...] At si oratores iverint, ut primum ad regem pervenient aliquem, statim ab hiis accedentur, qui unionis zelo ducuntur, instruenturque super omnibus, quomodo exordiendum sit, quomodo obiectionibus respondendum, qui favorabiles sint et qui adversantes ostendentur, cum hiis hoc modo, cum illis alio loqui oportere monebuntur ${ }^{30}$.

Il Pentalogus, riletto in questa chiave, si pone dunque come ricerca da parte del Piccolomini di un inserimento possibile nella realtà tedesca e nella corte, un inserimento che si resta però - come ho accennato - carico di incognite.

E questo lo si vede ancor di più se si considera in concreto quello che il Piccolomini sta facendo, il suo ruolo e la sua situazione effettiva. La posizione del Piccolomini in corte è in effetti una posizione ancora marginale, legata alle lettere che egli scrive, ora per Federico III, ora per il cancelliere Schlick, ora per altri membri della corte ${ }^{31}$. L'attività che egli svolge resta, cioè, si direbbe, limitata proprio alla sua funzione di segretario, che, come ricordava Federico III nel Pentalogus, ha solo il compito di scrivere le lettere che gli vengono affidate, senza tuttavia poter poi intervenire in modo attivo sulla politica regia, sulle sue direzioni e sui suoi obiettivi...

Ma, allora, qual è, quale può essere il significato di questo trattato? Che cosa rappresenta il Pentalogus? È una forma di lamentela, è l'espressione di una velleità personale, o dell'ideologia politica del Piccolomini?

Non credo. Credo piuttosto che il Pentalogus possa essere considerato come l'indicazione di un'esperienza che sta maturando, di quel che, come umanista, il Piccolomini è convinto di poter fare rispetto alla realtà tedesca, in una prospettiva che riflette il senso vivo delle difficoltà che egli incontra, ma anche, nello stesso tempo, la spinta costante a misurarsi, a confrontarsi con questo nuovo mondo. In questo senso, il Pentalogus ben rappresenta, in fondo, l'inizio effettivo e fruttuoso di quel rapporto che porterà, nel volgere di alcuni anni, al Piccolomini "cardinale tedesco".

30. Ivi, p. II8-I20.

3I. Si v. le lettere in Wolkan, Abt. I, Bd. II, Amtliche Schreiben. 
